\title{
Soft Computing Applications in Aircraft Sensor Management and Flight Control Law Reconfiguration
}

\author{
Marcel Oosterom, Member, IEEE, Robert Babuška, and Henk B. Verbruggen
}

\begin{abstract}
A sensor management system based on soft computing techniques has been developed and implemented in the flight control system of a small commercial aircraft. Unlike in the conventional sensor management system, the signals from sensors are assigned weights based on fuzzy membership functions and the consolidated signal is computed as a weighted average. This approach improves the quality of the consolidated signal and reduces transients due to sensor failures. This soft voting is extended to soft flight control law reconfiguration. In addition, a virtual sensor has been introduced as an arbitrator which enables the isolation of the failed sensor in the duplex operation and the detection of a sensor failure in the simplex operation. The effectiveness of the proposed methods is demonstrated by using an extensive simulation model of a small commercial aircraft, developed by airframe and control system manufacturers on the basis of an existing business jet. Furthermore, the system has been successfully evaluated and compared to standard techniques by means of pilot-in-the-loop simulations on the Research Flight Simulator of the National Aerospace Laboratory in The Netherlands. This application, developed within a Brite/EuRam research project, is characterized by the effective combination of novel soft computing techniques with standard, well proven methods of the aircraft industry. The properties of the conventional sensor management system have been retained, with the additional advantage that the quality of the consolidated signal is improved, the failure-induced transients are reduced, and the consolidated signal remains available up to the last valid sensor.
\end{abstract}

Index Terms-Aircraft control, analytical redundancy, controller reconfiguration, fuzzy logic, majority voting, sensor management, virtual sensor.

\section{INTRODUCTION}

$\mathbf{S}$ ENSOR management based on majority voting and point consolidation of like signals is a proven technology in modern fly-by-wire flight control systems [1]. The assumption is that the majority of like signals represent the truth and that any single dissimilar signal is the result of a failure. Such a signal must be disconnected as soon as the failure is detected. In the conventional approach, the decision whether a sensor has failed or not is crisp. In order to reduce the sensitivity of this decision to uncertainties like quantization and measurement noise, a properly adjusted threshold is used. This threshold is a compromise between two goals: the absence of false alarms and the ability to detect all possible failures within a certain time

Manuscript received March 26, 2001; revised April 22, 2002 This research was in part supported by the Brite/EuRam project "Affordable Digital Fly-by-wire Flight Control Systems for Small Commercial Aircraft" (ADFCS).

The authors are with Control Systems Engineering, Faculty of Information Technology and Systems, Delft University of Technology, NL-2600 GA Delft,

The Netherlands (e-mail: r.babuska@its.tudelft.nl).

Digital Object Identifier 10.1109/TSMCC.2002.801357. frame. This inevitably leads to a transient response during which the consolidated signal temporarily differs from the true value.

The soft sensor management system introduced in this paper maintains the key properties of the conventional sensor management system (majority voting) while improving its performance by applying fuzzy logic (FL) techniques. Although FL techniques have been implemented in other application domains, such as the process industry [2], [3], their application in flight control systems has not been extensively investigated yet. Using fuzzy logic, the decision whether a sensor has failed or not is no longer crisp. The sensor signals are assigned weights based on a cross-comparison of like signals by using fuzzy membership functions. The consolidated signal is then computed as a weighted average. Compared to the conventional management system, the soft management system intervenes at an earlier stage by reducing the weight of the suspected faulty sensor signal, while the failure declaration occurs at a later stage. This approach improves the quality of the consolidated signal with respect to its difference from the true value due to sensor failures. An attractive additional feature of this approach is the reduction of the transients due to sensor failures.

Furthermore, a virtual sensor is introduced in order to be able to identify failed sensors in the duplex operation and to detect a sensor failure in the simplex operation (which is not possible with the current sensor management systems). In the literature, many applications of analytical redundancy for fault detection and fault isolation in flight control systems have been reported [4]-[6], however, the use of virtual sensors in aerospace applications is novel.

The research described in this paper has been performed within the Brite/EuRam project "Affordable Digital Fly-by-wire Flight Control Systems for Small Commercial Aircraft" (ADFCS) [7]. The partners in this project are from the United Kingdom (BAE SYSTEMS), Israel (Israel Aircraft Industries, Israel Institute of Technology), Italy (Alenia Aerospazio, Centro Italiano Ricerche Aerospaziali), and The Netherlands (National Aerospace Laboratory, Delft University of Technology). The goal of the project is to investigate the application of fly-by-wire flight control systems (FBW FCSs) in small commercial aircraft in order to proliferate the proven benefits of the FBW technology, in terms of increased safety, commonality, productability, and maintainability. One part of the project investigates whether fuzzy and neural techniques can contribute to the development of a cost-effective FBW FCS. This application illustrates that the novel soft computing techniques can effectively be combined with standard well proven methods of the aircraft industry. 


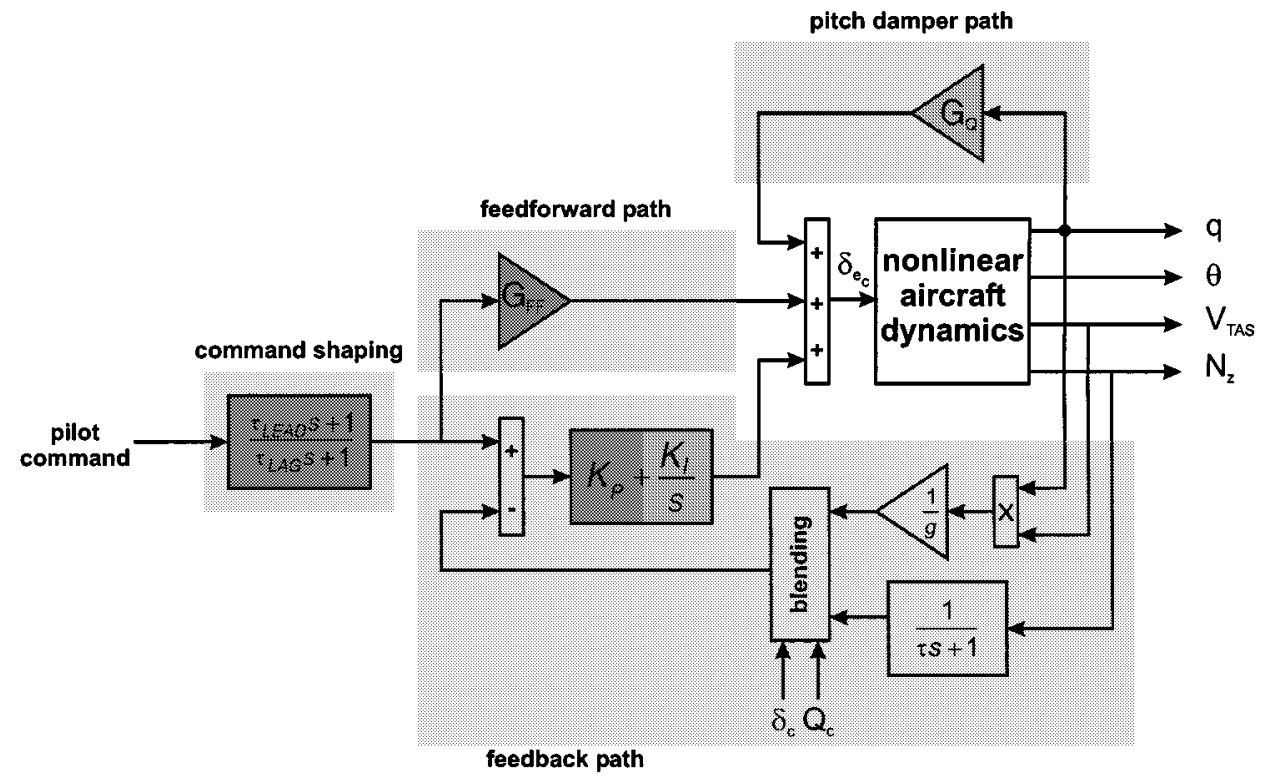

Fig. 1. Simplified structure of the longitudinal stability and control augmentation system. Input to the nonlinear model is the commanded elevator deflection $\delta_{e_{C}}$ [deg] and outputs are pitch rate $q$ [deg $\cdot \mathrm{s}^{-1}$ ], attitude $\theta$ [deg], true airspeed $V_{T A S}$ [knots], and normal acceleration $N_{z}[g]$. The blending in the feedback path is a function of the pitch column deflection $\delta_{c}[\mathrm{deg}]$ and dynamic pressure $Q_{c}[\mathrm{mbar}]$.

The paper is organized as follows. In Section II, the longitudinal flight control laws are briefly discussed in order to illustrate the implementation of the pitch rate and the normal acceleration feedback. The conventional sensor management system and the flight control law reconfiguration are discussed in Section III. Section IV introduces the sensor management system and flight control law reconfiguration based on soft computing, which is extended to include virtual sensors in Section V. Concluding remarks and future research are discussed in Section VI.

\section{LONGITUdinAL FLIGHT CONTROL LAWS}

In this section, a short description is given of that part of the longitudinal flight control laws (FCLs) that is relevant for the methods described in the remainder of this paper. The general objective of the FCLs integrated in a FBW system is to improve the flying qualities of the bare aircraft, in particular in the fields of stability, control and flight envelope protection [8]. From this point of view, the dynamics of the longitudinal short-period motion are of major importance.

In this paper, the Small Commercial Aircraft (SCA) model is used as a demonstrator [9]. The SCA model is a realistic aircraft model implemented in Matlab/Simulink ${ }^{\mathrm{TM}}$, based on an existing business jet aircraft. The SCA model is equipped with a stability and control augmentation system, which is part of the FCLs. A simplified structure of the longitudinal stability and control augmentation system is illustrated in Fig. 1. For the presented results, the pitch damper path and the feedback path are of main interest, since only the pitch rate and the normal acceleration sensor failures are taken into consideration. The command shaping path and the feedforward path are not addressed.

The pitch damper path augments the short-period damping of the bare aircraft through direct feedback of the pitch rate to the commanded elevator deflection. The "stiffness" of the closed-loop system is augmented through the feedback path by increasing the frequency of the short-period motion [10]. In

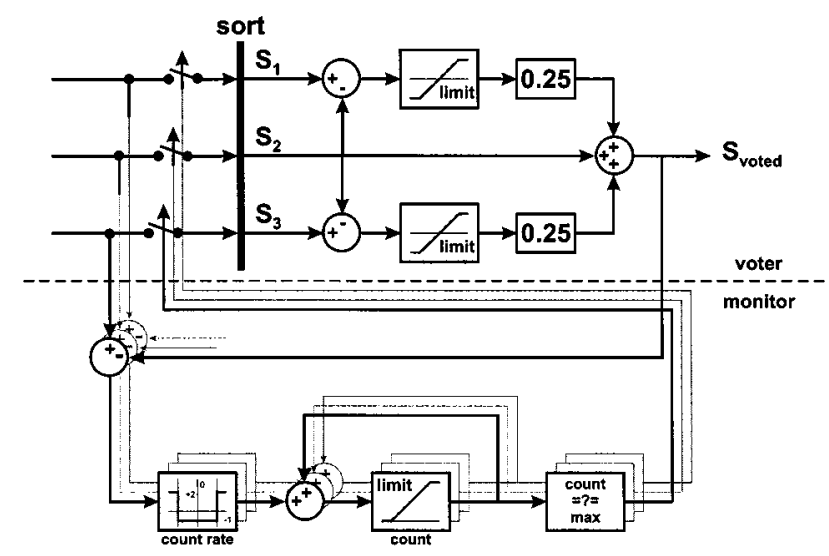

Fig. 2. Conventional triplex sensor management system (source: [1]).

order to be able to augment the stiffness, feedback of the angleof-attack $\alpha$ is required (the frequency of the short-period motion is a function of the aerodynamic coefficient $C_{m_{\alpha}}$ ). Since the $\alpha$-sensor is not accurate enough to be used for control, this signal is reconstructed by using a blending of the pitch rate $q$ and the normal acceleration $N_{z}$. This blending is a function of the pitch column deflection $\delta_{c}$ (pilot input) and the dynamic pressure $Q_{c}$ (function of airspeed and altitude). With the pitch column centered, the feedback signal is only influenced by the pitch rate. The same holds for large pitch column deflections at low dynamic pressure. The normal acceleration is entering the feedback signal for large pitch column deflections at medium and high dynamic pressure.

\section{Conventional Sensor Management AND FCL RECONFIGURATION}

Each signal is measured independently by a number of sensors. The sensor management system has two tasks, namely the computation of a consolidated signal from these measurements 

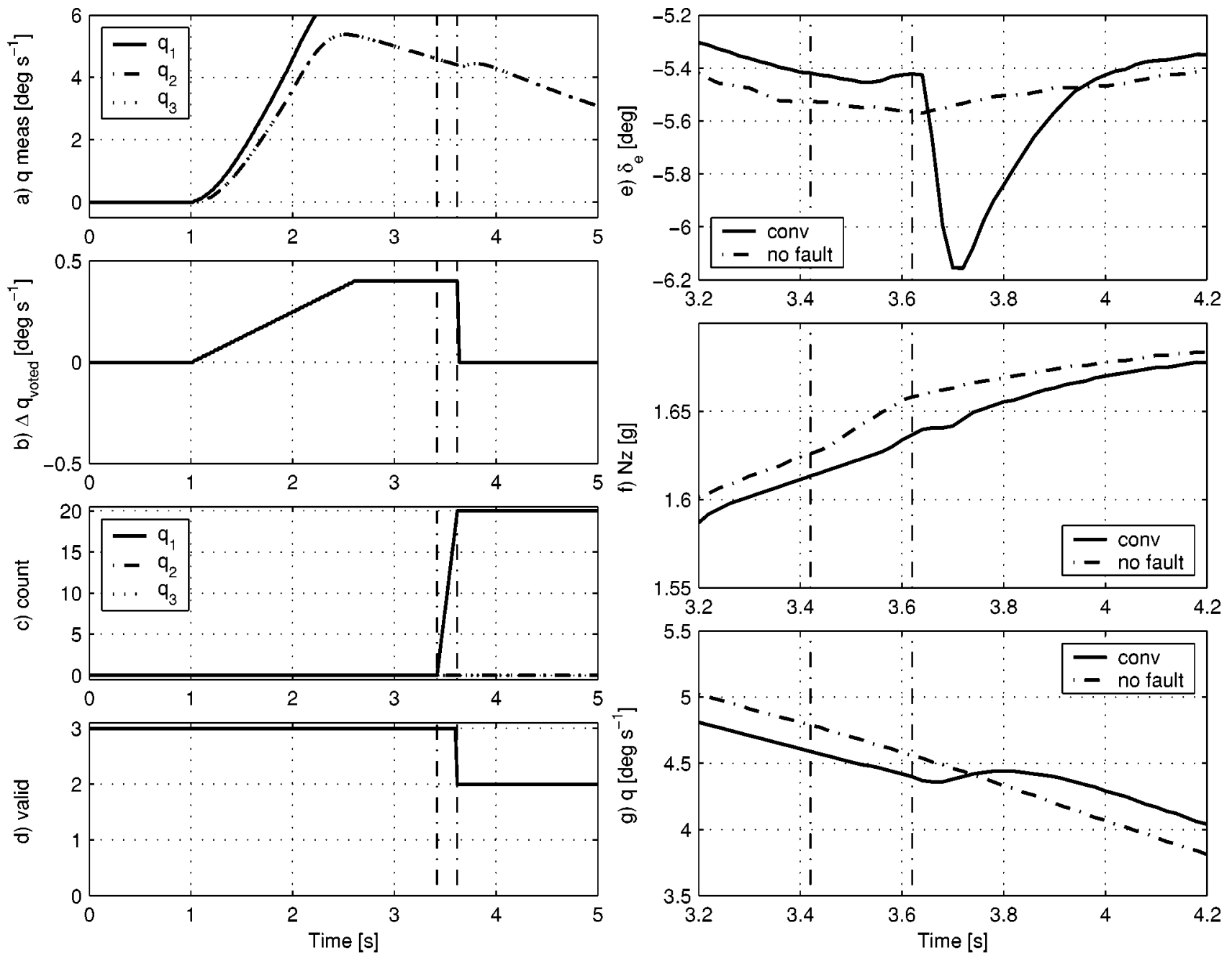

Fig. 3. Conventional sensor management: drift failure of a pitch rate sensor. Figures e-g are zoomed in on the failure-induced transients.

(voting) and the validation of each of the sensors (monitoring). The consolidated (or voted) signal is fed to the flight control computer and at the same time serves as a reference for sensor validation.

\section{A. Conventional Voting/Monitoring Scheme}

The redundancy level for each signal, i.e., the number of redundant sensors in normal operation, is related to the failure probability of the sensor and the consequence of losing the corresponding signal. For example, the consequence of losing the pitch rate signal is a catastrophic failure. The probability of a catastrophic failure must be less than $10^{-9}$ per hour of flight. If the failure probability of a pitch rate sensor is $10^{-4}$ per hour of flight, the third consecutive like sensor failure may result in a catastrophic failure. A sensor failure in the duplex operation (two sensor signals available) results in losing the signal, since the conventional voting/monitoring scheme is not able to identify the failed sensor in this case. This implies that four pitch rate sensors need to be implemented in order to meet the requirements regarding the probability of a catastrophic failure. This is called the quadruplex system. Similarly, we have the triplex, duplex and simplex voter for three, two and one physical sensor, respectively.
In order to keep the presentation simple, the triplex voter will be used to explain the conventional voting/monitoring philosophy (see Fig. 2). The three sensor signals are first sorted from the largest value to the smallest one. The mid-value signal is taken as a reference and the two extreme-value signals are limited in their deviation from the mid-value signal. When the limits are not invoked, the voted (consolidated) signal is given by

$$
\begin{aligned}
S_{\text {voted }} & =S_{2}+0.25 \cdot\left(S_{1}-S_{2}\right)+0.25 \cdot\left(S_{3}-S_{2}\right) \\
& =0.25 \cdot\left(S_{1}+2 S_{2}+S_{3}\right) .
\end{aligned}
$$

For two valid signals, a duplex voter is used, and the voted signal is a simple average.

The monitor compares each of the three sensor signals $S_{i}$ with the consolidated signal $S_{\text {voted }}$. If the absolute difference is smaller than a predefined threshold $\Delta$, the monitor count is decreased by one, otherwise it is increased by two:

$$
\begin{aligned}
& \text { If } \left.\left|S_{i}-S_{\text {voted }}\right| \leq \Delta \text { then (count rate }\right)_{i}=-1 \\
& \text { If }\left|S_{i}-S_{\text {voted }}\right|>\Delta \text { then }(\text { count rate })_{i}=+2 .
\end{aligned}
$$

The updated count value is bounded between zero and the failure declaration value. If the count value has reached the failure declaration value, a failure is declared and the signal is latched (see Fig. 2). 

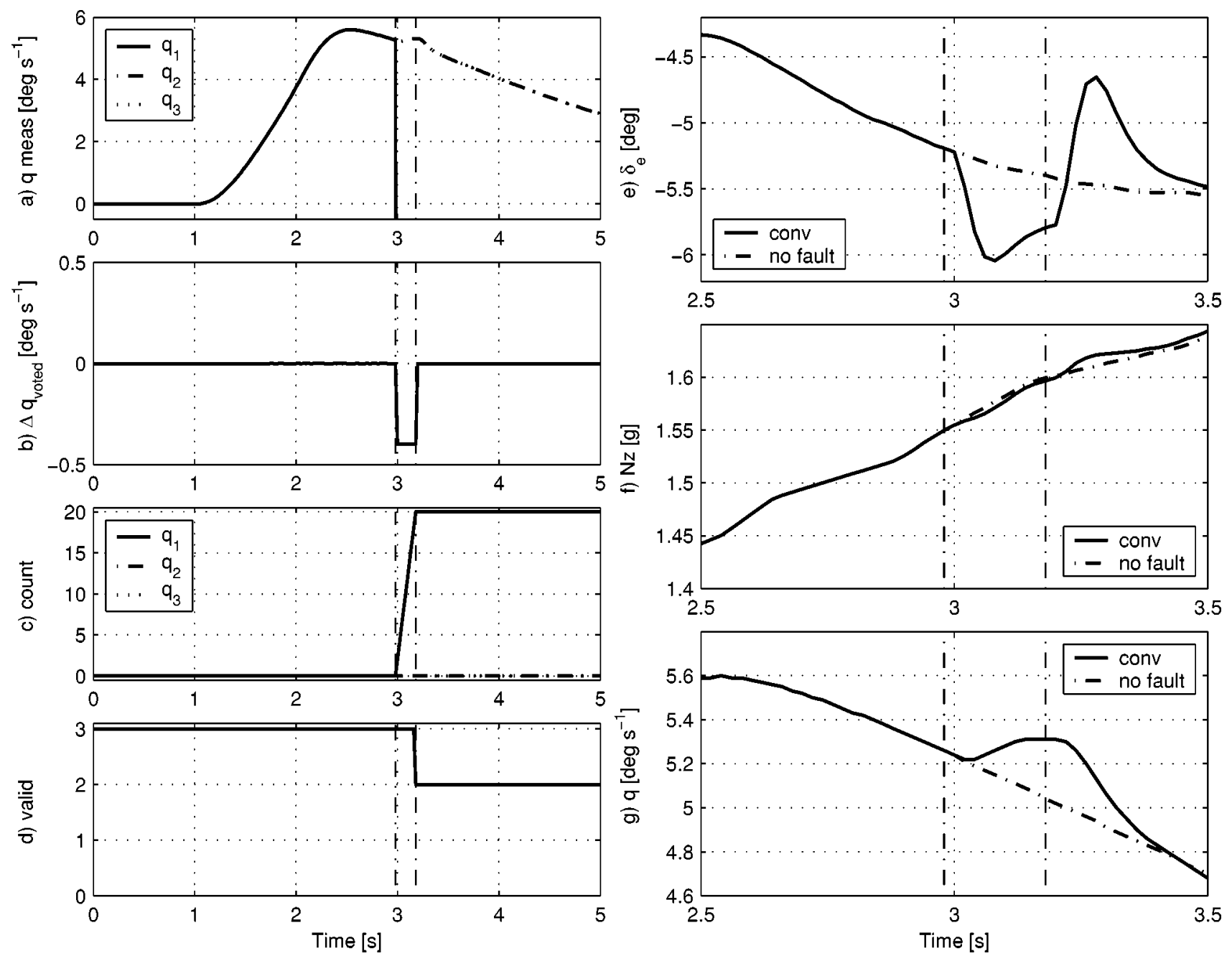

Fig. 4. Conventional sensor management: cutoff failure of a pitch rate sensor. Figures e-g are zoomed in on the failure-induced transients.

The logic in the conventional sensor management system is such that a failed sensor output continues to contribute to the voted signal until it is latched. This results in transients and discontinuities in the voted signal, which is illustrated in two nonlinear, closed-loop simulation examples using the SCA Matlab/ Simulink ${ }^{\mathrm{TM}}$ model.

\section{B. Simulation Examples}

For both simulations, the initial condition is a straight and level flight at a Mach number of $M=0.75$ and an altitude of $h=40 \mathrm{kft}$, which is the cruise flight condition for the SCA model. The pilot input is a block-shaped input of the maximum positive column deflection starting at $t=1 \mathrm{~s}$ and lasting for 6 s. During this maneuver, the normal acceleration signal is partly contributing to the feedback path. The corresponding time histories of the first simulation example are illustrated in Fig. 3. At $t=1 \mathrm{~s}$, a drift failure of $1 \mathrm{deg} \cdot \mathrm{s}^{-2}$ occurs in one of the pitch rate sensors [Fig. 3(a)]. Fig. 3(b) shows the difference $\Delta q_{v o t e d}$ between the voted signal and the true pitch rate. Due to the drift failure, the voted signal diverges from the true pitch rate until the contribution of the failed sensor output is limited and the mismatch remains constant. When the difference between the failed sensor $\left(q_{1}\right)$ and the voted signal $\left(q_{v o t e d}\right)$ exceeds the monitor threshold, the monitor count rate increases from -1 to +2 (denoted by the first vertical dash-dotted line). When the monitor count [Fig. 3(c)] reaches the failure declaration value (denoted by the second dash-dotted vertical line) the signal is latched and the number of valid signals reduces to two [Fig. 3(d)]. The faulty contribution of $q_{1}$ is omitted instantaneously, which results in an undesirable discontinuity in the consolidated signal [Fig. 3(b)]. The resulting transients in the elevator deflection [Fig. 3(e)], the normal acceleration [Fig. 3(f)], and the true pitch rate [Fig. 3(g)] signals are evident (solid line) compared to the fault free case (dash-dotted line).

The second simulation example illustrates a cutoff sensor failure which occurs at $t=3 \mathrm{~s}$ in one of the pitch rate sensors [Fig. 4(a)]. Due to the abrupt nature of the sensor failure, discontinuities in the voted signal occur both when the failure is inserted and when the corresponding signal is latched [Fig. 4(b)]. Again the behavior of the voted signal is undesirable, since it is by no means representing the behavior of the true signal. The transients in the elevator deflection [Fig. 4(e)], the normal acceleration [Fig. 4(f)] and the pitch rate [Fig. 4(g)] are evident (solid line), especially when compared to the fault free case (dash-dotted line). 

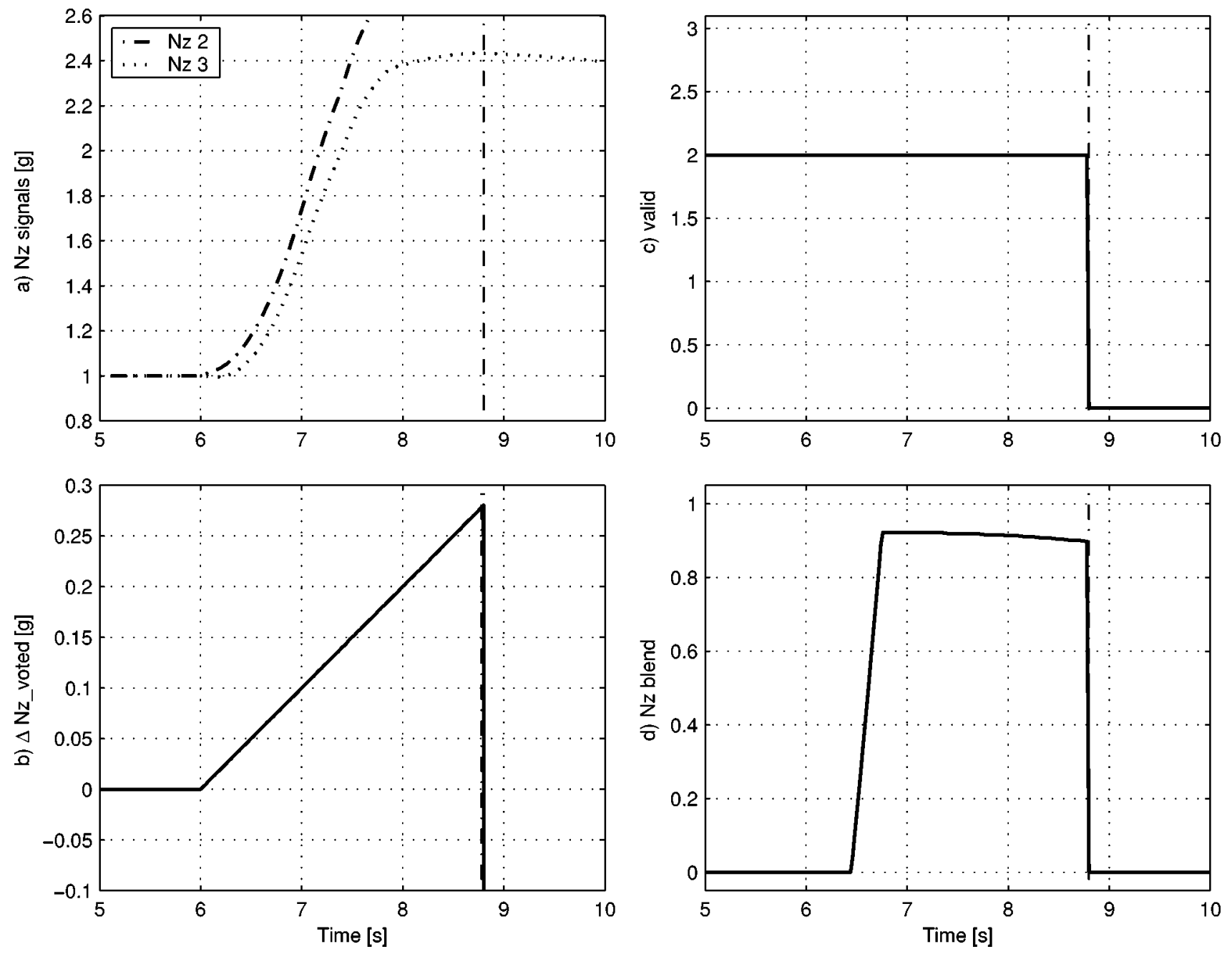

Fig. 5. Conventional sensor management: a drift failure of the second normal acceleration sensor.

\section{Flight Control Law Reconfiguration}

The voted signal in the duplex operation is computed as the average of the two sensor signals. If a sensor fails in the duplex operation, the majority voting principle can no longer be used to identify the failed sensor. As soon as the difference between these two signals exceeds a certain threshold, both sensors are declared invalid and the FCS reconfigures to not using this particular signal.

In Fig. 5(a), a drift failure of the second normal acceleration sensor is simulated [Fig. 5(a)]. The voted signal is the average of the two valid signals. The initial condition is a straight and level flight at a Mach number of $M=0.70$ and an altitude of $h=25 \mathrm{kft}$. This flight condition is selected to increase the contribution of the normal acceleration signal in the feedback path. The pilot input is a block-shaped input of maximum positive column deflection starting at $t=6 \mathrm{~s}$ and lasting for $6 \mathrm{~s}$. When the difference between the two sensor signals exceeds the threshold, the monitor count of both sensor signals is set to the failure declaration value instantaneously and both input signals are latched [Fig. 5(c)]. At this point the consolidated signal is no longer available and the FCS reconfigures to not using this signal [Fig. 5(d)]. This implies that only the pitch rate signal is used in the feedback path for the entire range of the admissible column deflection and dynamic pressure.

The normal acceleration signal is used to demonstrate the conventional management system in the case of a sensor failure in duplex operation, since there is no FCL reconfiguration available for the loss of the pitch rate signal in the SCA model. As mentioned above, the loss of the pitch rate signal is a catastrophic failure.

The above examples have demonstrated the main shortcomings of the conventional sensor management approach-the failure-induced discontinuities in the consolidated signal and the inability to identify sensor failures in duplex operation and/or to detect a sensor failure in simplex operation. A Fuzzy Logic (FL) approach can be used to improve the conventional sensor management system without changing the basic concept of majority voting. This is described in the remainder of this paper.

\section{Sensor Management and Flight Control Law RECONFIGURATION BASED ON SOFT COMPUTING}

In this section, we focus on reducing, or even eliminating, the transients in the voted signal due to sensor failures. A new sensor management scheme is introduced, which makes use of fuzzy logic. 


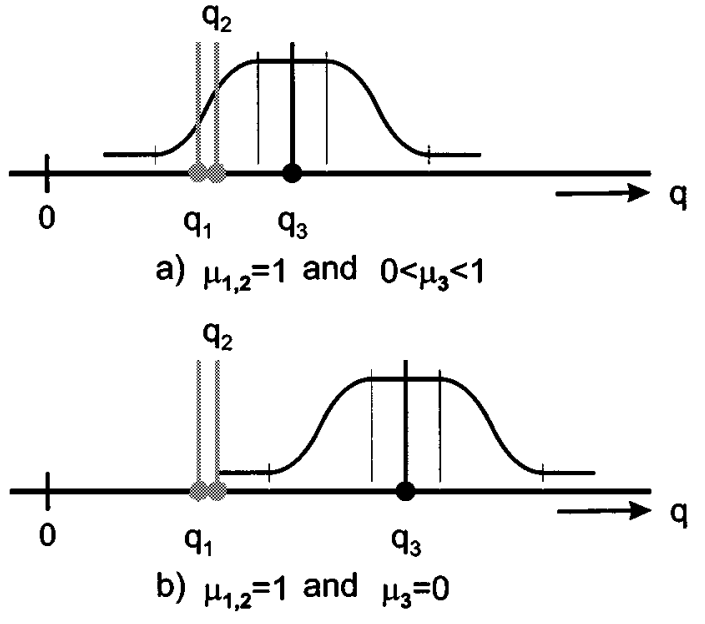

Fig. 6. Soft voting in the triplex operation. The current value of each sensor signal forms the center of its corresponding membership function, which is used to determine the membership degree of this sensor signal.

\section{A. Soft Voting/Monitoring Scheme}

The soft voter is different from the conventional voting scheme in the sense that each input signal is assigned a weight, and the consolidated signal is the weighted average of the input signals

$$
S_{v o t e d}=\sum_{i=1}^{n} w_{i} S_{i}
$$

where $w_{i}$ denotes the weight assigned to the $i$ th input signal $S_{i}$ and $n$ denotes the number of valid sensors. The weight $w_{i}$ is the normalized membership degree $\mu_{i}$

$$
w_{i}=\frac{\mu_{i}}{\sum_{j=1}^{n} \mu_{j}}
$$

where $0 \leq \mu_{i} \leq 1$. The computation of the membership degree $\mu_{i}$ is explained by using Fig. 6 .

The current value of each signal forms the center of its corresponding membership function. The membership degree of the signal is the largest membership degree of the remaining valid signals according to this membership function

$$
\mu_{i}=\max _{i \neq j}\left(\mu_{i}\left(q_{j}\right)\right) \text {. }
$$

In Fig. 6(a), the membership function of $q_{3}$ is illustrated. With respect to this membership function, $q_{1}$ has a membership degree of $\mu_{3}\left(q_{1}\right)=0.35$ and $q_{2}$ has a membership degree of $\mu_{3}\left(q_{2}\right)=0.65$. This implies that $q_{3}$ has a membership degree of

$$
\mu_{3}=\max \left(\mu_{3}\left(q_{1}\right), \mu_{3}\left(q_{2}\right)\right)=0.65 \text {. }
$$

Clearly, the majority voting concept of the conventional sensor management system is also used in the soft sensor management system. The signal $q_{3}$ is not in agreement with the signals $q_{1}$ and $q_{2}$, and therefore its weight in the voted signal is reduced. In Fig. 6(b), the discrepancy between signal $q_{3}$ and the signals $q_{1}$ and $q_{2}$ is further increased. The corresponding membership degree is now reduced to $\mu_{3}=0$.
Both the conventional and the soft voting scheme are based on majority voting. The major difference is the way the like sensor signals contribute to the consolidated signal. In the conventional voting scheme, the contribution of a faulty signal is limited, while in the soft voting scheme its weight is reduced. The implementation of the soft voting scheme is illustrated in Fig. 7 for a triplex sensor system. The vector of like signals is split (demux) and sorted (sort). The membership degrees are computed according to (3), put back in the original order (desort) and combined again in a vector (mux). The voted signal is then computed according to (1) and (2).

In the monitor part, the count rate of the $i$ th signal is the following function of its corresponding membership degree $\mu_{i}$ :

$$
\begin{aligned}
& \text { If } \mu_{i}=1 \text { then }(\text { count rate })_{i}=-1 \text {; } \\
& \text { If } 0<\mu_{i}<1 \text { then }(\text { count rate })_{i}=0 \text {; } \\
& \text { If } \mu_{i}=0 \text { then }(\text { count rate })_{i}=+2 .
\end{aligned}
$$

The main difference from the conventional monitoring scheme is that here the monitor count rate is not a function of the difference between the $i$ th sensor reading and the voted signal, but a function of the difference between the $i$ th sensor reading and the other like sensor readings. The count rate of the $i$ th sensor signal becomes positive when the corresponding weight in the voted signal is equal to zero $\left(w_{i}=0\right)$, therefore no transients occur once the failure is declared on the $i$ th sensor and the corresponding signal is latched. This is illustrated with the help of two closed-loop simulation examples.

\section{B. Simulation Examples}

The setting is identical to the simulation examples discussed in Section III-B except for the sensor management system.

The time histories of the first simulation example are given in Fig. 8. At $t=1 \mathrm{~s}$, a drift failure of $1 \mathrm{deg} \cdot \mathrm{s}^{-2}$ occurs [Fig. 8(a)]. Fig. 8(b) shows the difference between the voted signal and the true pitch rate $\Delta q_{v o t e d}$. Due to the drift failure the voted signal diverges from the true pitch rate until the weight of the failed sensor output is reduced to zero [Fig. 8(c)] and the voted signal is again equal to the true value (not taking into account uncertainties such as quantization, sensor noise, etc.). One can see that the voted signal is smoother than in the conventional sensor management case. By this time the monitor count rate is increased from $-1\left(\mu_{1}=1\right)$ to $0\left(0<\mu_{1}<1\right)$ and from 0 to $+2\left(\mu_{1}=0\right)$. When the monitor count reaches the failure declaration value [Fig. 8(d)] the signal is latched, and the number of valid signals reduces to two (denoted by the second dash-dotted line). As the weight of the corresponding signal is equal to zero at the moment of the failure declaration, no transients occur; see the solid lines of the elevator deflection [Fig. 8(e)], normal acceleration [Fig. 8(f)], and the true pitch rate [Fig. 8(g)]. For comparison, the time histories of the simulations of the fault free case (dash-dotted line) and conventional voting/monitoring case (dotted line) are also included in Fig. 8(e)-(g).

The second simulation example is illustrated in Fig. 9. At $t=3 \mathrm{~s}$, a cutoff sensor failure occurs [Fig. 9(a)]. Due to the abrupt nature of the sensor failure, the weight of the failed signal output becomes zero immediately [Fig. 9(c)] and therefore there are no transients in the elevator deflection [Fig. 9(e)], the normal acceleration [Fig. 9(f)], and the pitch rate [Fig. 9(g)] signals. 


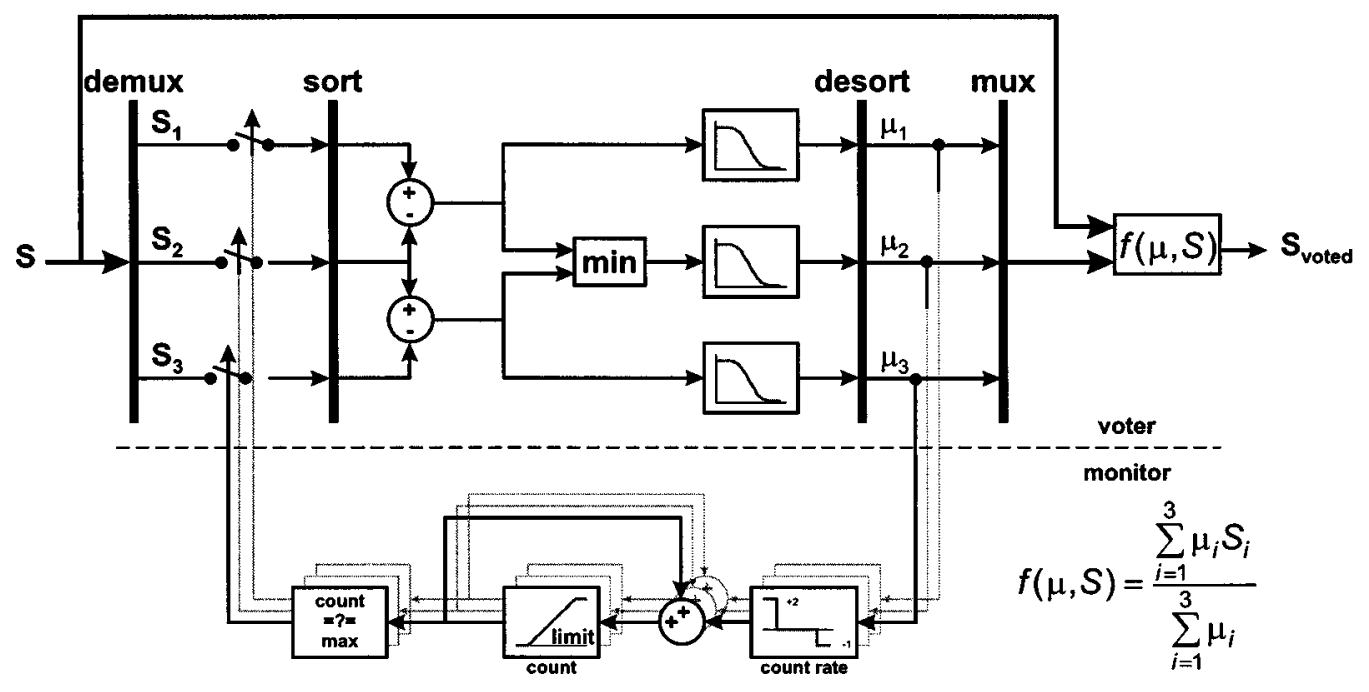

Fig. 7. Soft triplex sensor management system.
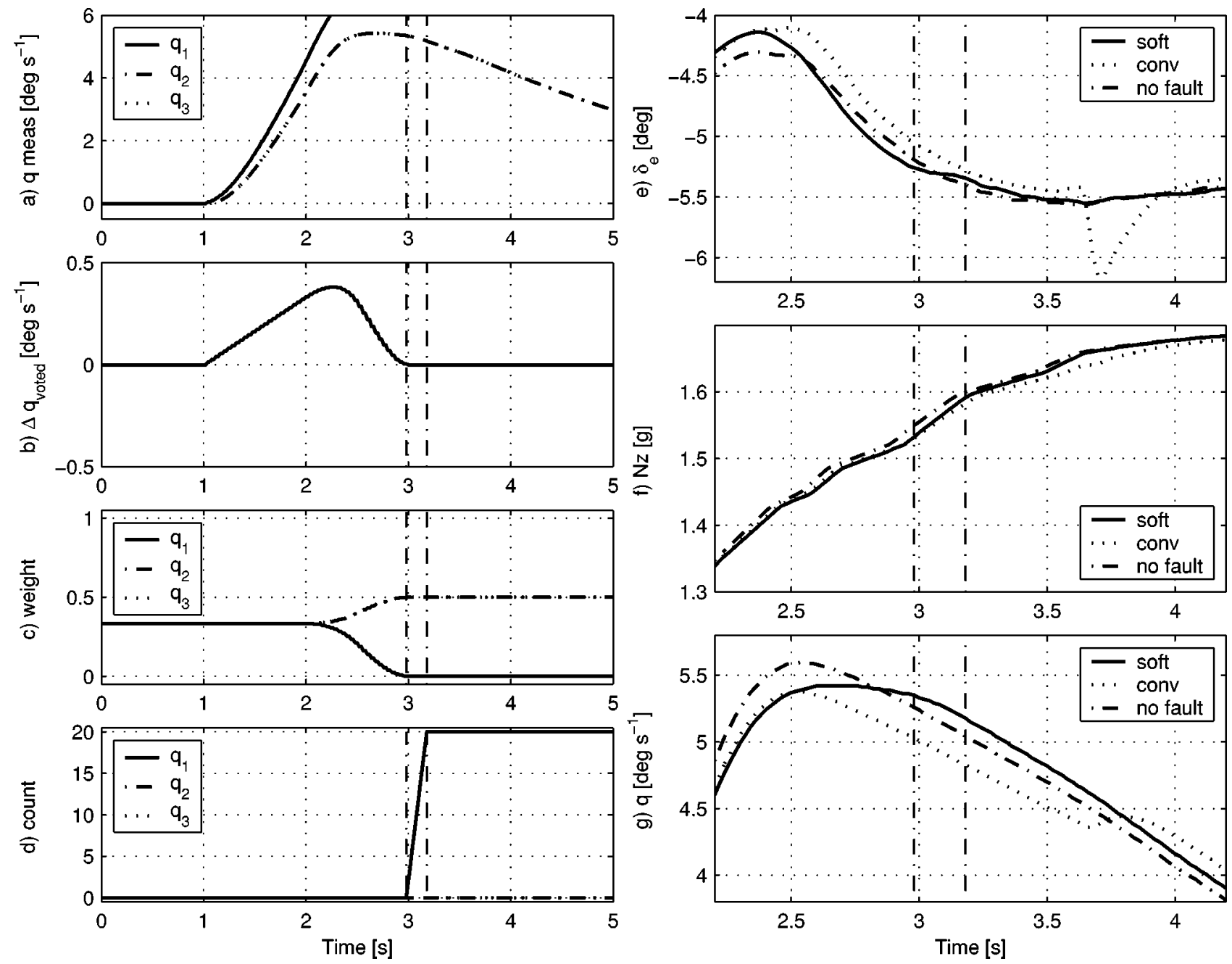

Fig. 8. Soft sensor management: drift failure of a pitch rate sensor. (e)-(g) Zoomed in on the failure-induced transients.

The closed-loop transients due to sensor failures are not of the same order for each signal. From Fig. 1, it can be seen that the pitch rate is fed back through a proportional gain in the pitch damper path and through a proportional and an integral gain in the feedback path. In the feedback path the normal acceleration is fed back in a similar way. However, discontinuities in the voted normal acceleration signal are suppressed by the low-pass filter in the normal acceleration feedback path (Fig. 1). Closed-loop transients are therefore less evident. For this reason the pitch rate signal is used to demonstrate this additional benefit of the sensor management system based on soft computing techniques. 

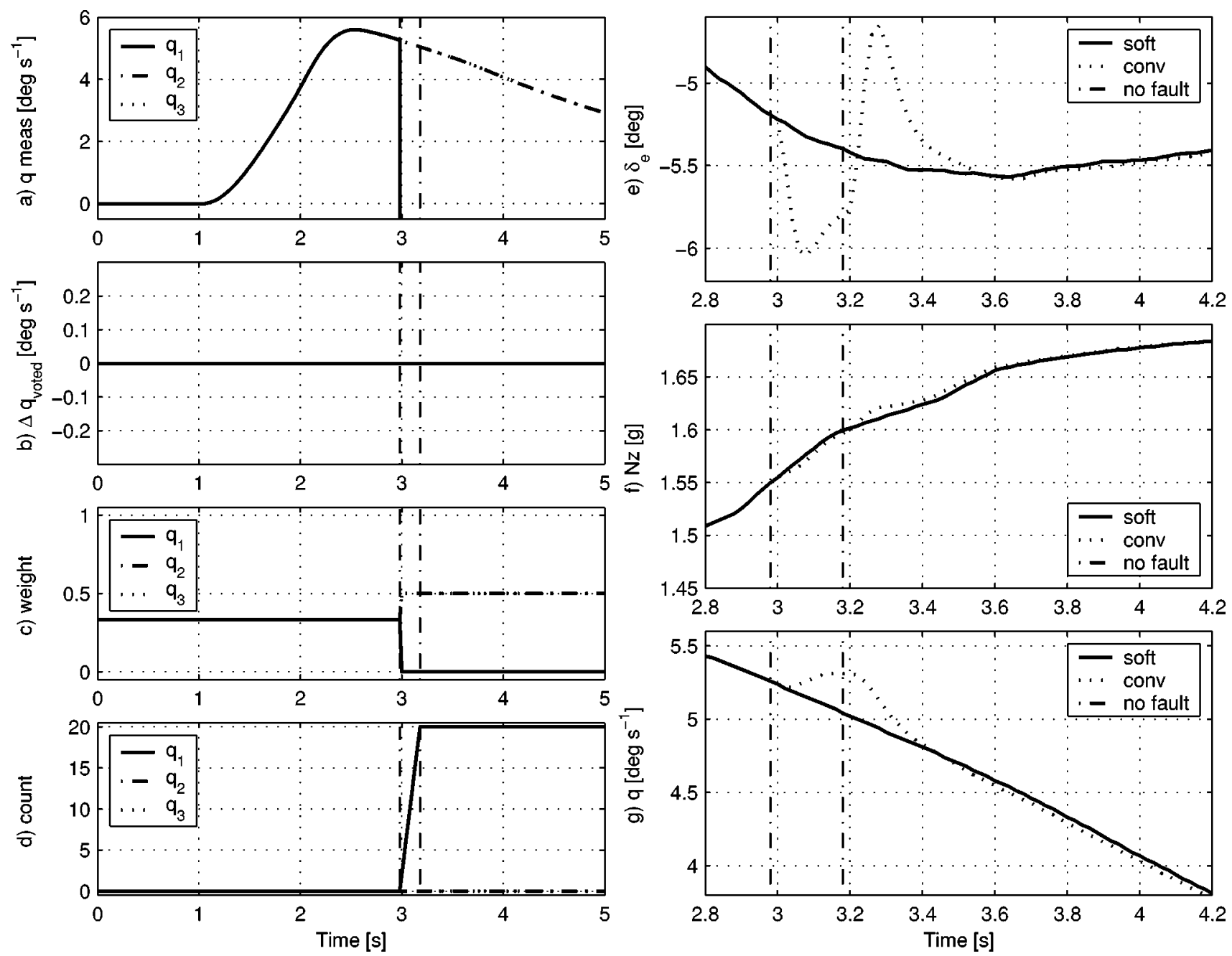

Fig. 9. Soft sensor management. Cutoff failure of a pitch rate sensor. (e)-(g) Zoomed in on the failure-induced transients.

\section{Flight Control Law Reconfiguration}

The soft voting logic is extended to soft flight control law reconfiguration. Also here, the voted signal is computed as the average of the two sensor signals. Both sensor signals automatically have the same membership degree, and are therefore equally weighted in the consolidated signal. However, their mutual membership degree is multiplied with the contribution of the normal acceleration signal in the feedback path as well. The blending between the pitch rate and the normal acceleration signals in the feedback path (see Fig. 1) is now a function of the column deflection $\delta_{c}$ and the dynamic pressure $Q_{c}$ multiplied by the maximum membership degree of the normal acceleration signals. When the difference between the two signals is such that their mutual membership degree becomes equal to zero, the flight control laws are already reconfigured to not using the normal acceleration signal in the feedback path.

This is illustrated in Fig. 10, where the time histories of a simulation of a drift failure of the second normal acceleration sensor are illustrated [Fig. 10(a)]. The voted signal is the average of the two input signals. During the maneuver, the signal in the feedback path is for $90 \%$ derived from the normal acceleration signal [Fig. 10(d)]. This is reduced to zero due to the growing discrepancy between the two valid normal acceleration sensor signals.
By the time the $N z$ signal is no longer available [Fig. 10(b)], the FCLs are reconfigured to not using this signal [Fig. 10(d)].

\section{Discussion}

The voter has the task to provide a good signal for control purposes based on the premise that most, if not all, of the available inputs are healthy. The monitor has the task to detect and identify any "unhealthy" signals that may cause a problem with the continued safe control of the aircraft.

In principle, the conventional and the soft sensor management system are much alike. The soft sensor management system is a weighted implementation of the conventional sensor system, retaining all the benefits of this system.

The performance of the soft sensor management system is always better or equal to that of the conventional sensor management system with respect to the behavior of the consolidated signal due to sensor failure and its deviation from the true value, as has been demonstrated by simulation examples. This benefit is most evident for cutoff failures. The worst case sensor failure for the soft sensor management system is a step-like sensor failure that does not result in a membership degree of the corresponding signal that is equal to zero. Only in this case a discontinuity in the consolidated signal occurs. 

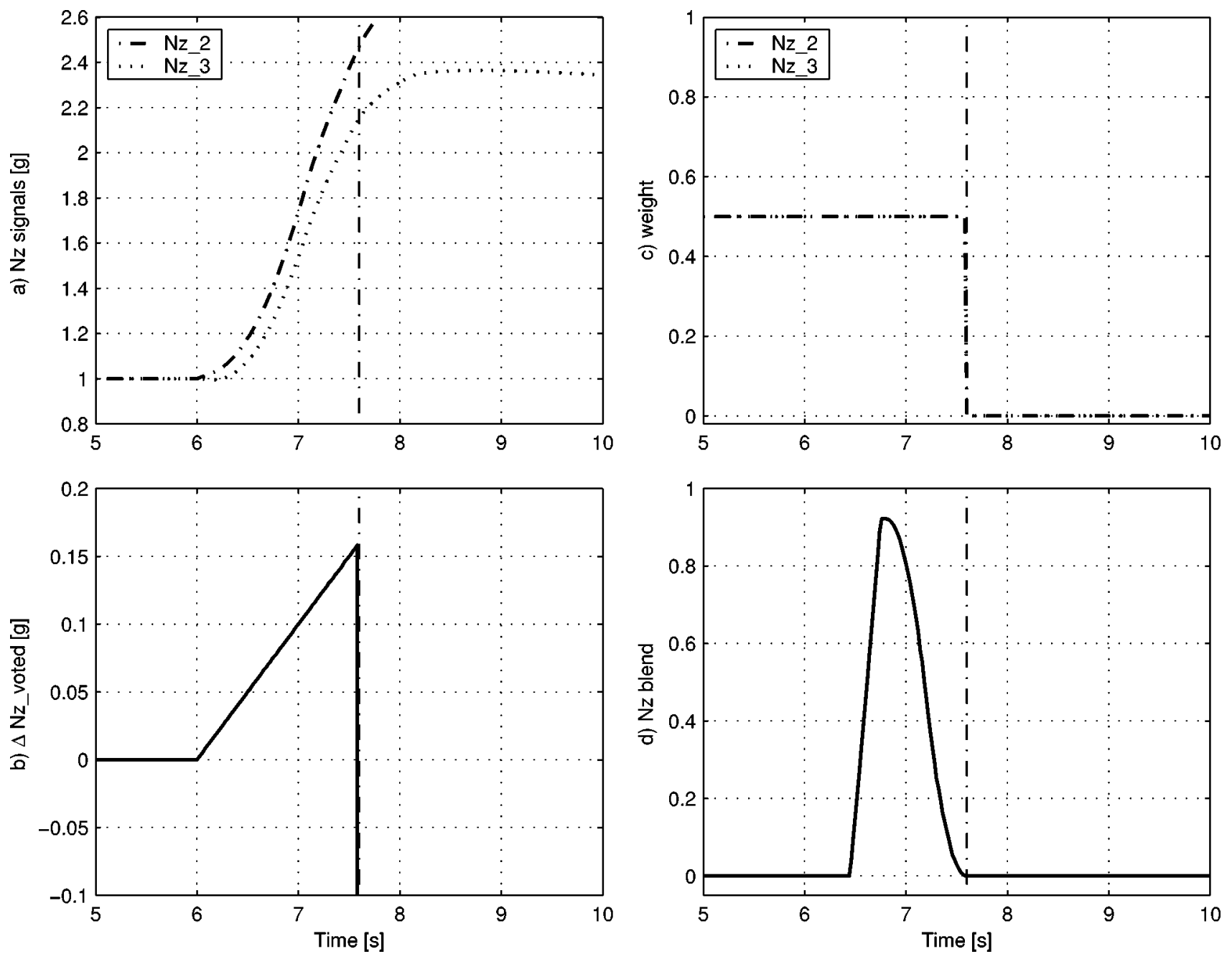

Fig. 10. Soft sensor management: a drift failure of the second normal acceleration sensor.

The conventional voting/monitoring system has two separate crisp thresholds, one to limit the contribution of a suspected faulty sensor signal and one for the failure declaration. The selected thresholds are a compromise between two goals: the absence of false alarms and the ability to detect all possible failures within a short time frame. The latter is important to minimize the effect of a sensor failure. This inevitably leads to a transient response during which the consolidated signal temporarily differs from the true value. Although it is possible to reduce transients by introducing filters, the soft sensor management is a more direct solution to this problem. In the soft sensor management system, the compromise between false alarms and the ability to detect sensor failures within a certain time frame is avoided by introducing a fuzzy threshold. Through the fuzzy threshold objectives of no false alarms and the minimization of the effect of a sensor failure are well separated. When the failure declaration procedure is activated, the weight of the corresponding sensor signal is equal to zero and the negative impact of the suspected faulty sensor is already taken care of. The soft sensor management system is representative for the real life situation of decision making process in general, and fault detection and isolation in particular. As transients are reduced or even removed, the tuning of the membership function parameters is only driven by the sensor characteristics. Expensive sensors are more accurate and may need narrower membership functions. The additional computation due to the soft sensor management system is considered to be negligible. The thresholds used in the simulation examples were selected by the authors such that the characteristics of both voting/monitoring systems become clear. The crisp threshold in the conventional voting system is in between the upper and lower bound of the fuzzy threshold of the soft voting/monitoring system.

Information on the membership degree can be used for maintenance purposes. If a sensor has regularly a membership degree lower than one, this is an indication that something is wrong and that the corresponding sensor needs to be replaced.

\section{VIRTUAL SENSORS AND DYNAMIC THRESHOLDS}

The conventional sensor management system works well down to two signals, where any discrepancy can no longer be related to a "majority". In this instance, the system will either reject both signals and reconfigure to not using this information, or, for essential data, a simple average will be used as the best compromise. However, there is additional information available that can be used to identify the failed sensor in the duplex 
operation and to detect a failure in the simplex operation. This additional information can be employed to estimate the signal of interest, by using the so-called virtual sensor. Monitoring of the hardware sensor(s) in the duplex and simplex operation is then performed by comparison with the virtual sensor output.

\section{A. Analytical Redundancy}

In the literature, many applications of analytical redundancy for fault detection and isolation in flight control systems have been reported. Most frequently applied are linear observer-based techniques [4], [11], parity-space methods [5], [12]-[14], and parameter-estimation schemes [6], [15]. The use of nonlinear virtual sensors in aerospace applications has not been extensively investigated yet, although this technique has been successfully applied in other domains like process control and engine control [16], [17].

In [18] we proposed a virtual sensor of the Takagi-Sugeno (TS) fuzzy model type [19] that uses dissimilar consolidated sensor readings as inputs to estimate the normal acceleration. The structure of the virtual sensor, based on the physical model of the aircraft, is as follows:

$$
N_{z}=\frac{1}{g} \cdot(\dot{w}-u \cdot q+g \cdot \cos \theta \cdot \cos \phi)
$$

where $u$ denotes the forward velocity, $w$ the downward velocity, $q$ the pitch rate, $\theta$ the pitch attitude, $\phi$ the bank angle and $g$ the gravitational acceleration. The time derivative of the downward velocity can locally be described by the following linear expression

$$
\dot{w}=Z_{u} u+Z_{w} w+Z_{q} q+Z_{\theta} \theta+Z_{\delta_{e}} \delta_{e}+Z_{\delta_{s}} \delta_{s}
$$

where $\delta_{e}$ denotes the elevator deflection and $\delta_{s}$ the stabilizer deflection. The parameters $Z_{u}$ through $Z_{\delta_{s}}$ are the so-called aerodynamic derivatives. However, due to the nonlinearity of the aircraft, the aerodynamic derivatives are not constant, but depend on the Mach number $M$, the dynamic pressure $Q_{c}$ and the angle-of-attack $\alpha$. Taking this dependence into account, the structure of the $\dot{w}$ estimator becomes

$$
\begin{aligned}
\dot{w}_{T S}= & Z_{0}\left(M, Q_{c}, \alpha\right)+Z_{u}\left(M, Q_{c}, \alpha\right) \cdot u+Z_{w}\left(M, Q_{c}, \alpha\right) \cdot w \\
& +Z_{q}\left(M, Q_{c}, \alpha\right) \cdot q+Z_{\theta}\left(M, Q_{c}, \alpha\right) \cdot \theta \\
& +Z_{\phi}\left(M, Q_{c}, \alpha\right) \cdot \phi+Z_{\delta_{e}}\left(M, Q_{c}, \alpha\right) \cdot \delta_{e} \\
& +Z_{\delta_{s}}\left(M, Q_{c}, \alpha\right) \cdot \delta_{s} .
\end{aligned}
$$

Note the additional offset $Z_{0}\left(M, Q_{c}, \alpha\right)$, introduced to compensate for steady-state values of the input signals. The term $Z_{\phi}\left(M, Q_{c}, \alpha\right) \cdot \phi$ was added after the first flight simulator evaluation, because the virtual sensor was unable to estimate the downward acceleration correctly during high bank maneuvers.

The nonlinear functions $Z_{i}\left(M, Q_{c}, \alpha\right)$, representing the aerodynamic derivatives, are approximated by TS fuzzy rules of the following form:

1) If $M$ is $L O W$ and $Q_{c}$ is $L O W$ and $\alpha$ is $L O W$

then $Z_{0_{1}}=Z_{0_{1_{0}}}+Z_{0_{1_{M}}} M+Z_{0_{1_{Q_{c}}}} Q_{c}+Z_{0_{1_{\alpha}}} \alpha$

2) If $M$ is $L O W$ and $Q_{c}$ is $L O W$ and $\alpha$ is $H I G H$ then $Z_{0_{2}}=Z_{0_{2_{0}}}+Z_{0_{2_{M}}} M+Z_{0_{2_{Q_{c}}}} Q_{c}+Z_{0_{2_{\alpha}}} \alpha$

3) If $M$ is $H I G H$ and $Q_{c}$ is $H I G H$ and $\alpha$ is $H I G H$ then $Z_{0_{s}}=Z_{0_{s_{0}}}+Z_{0_{s_{M}}} M+Z_{0_{s_{Q_{c}}}} Q_{c}+Z_{0_{s_{\alpha}}} \alpha$.

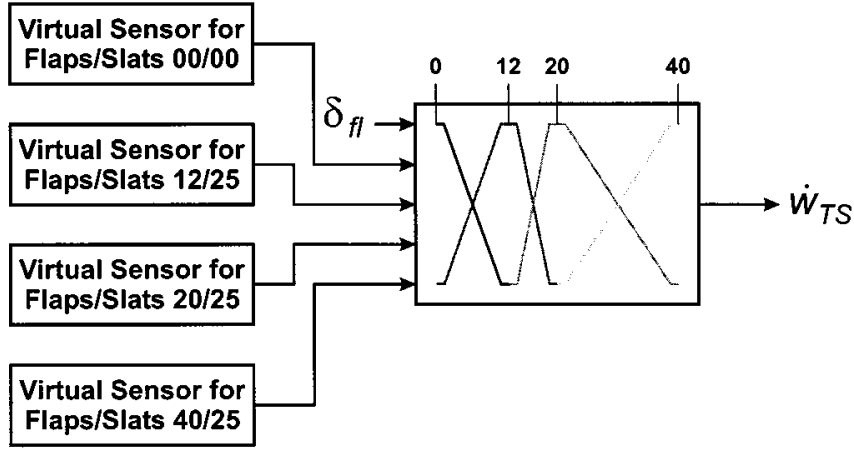

Fig. 11. Architecture of the virtual sensor.

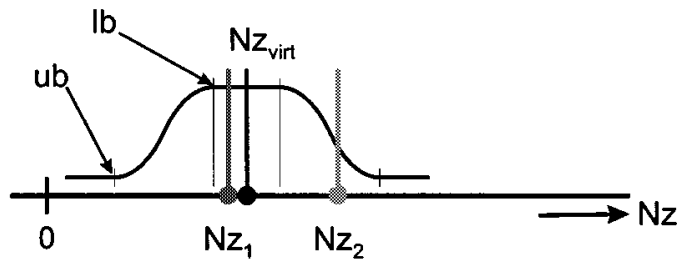

a) $\mu_{1}=1$ and $0<\mu_{2}<1$

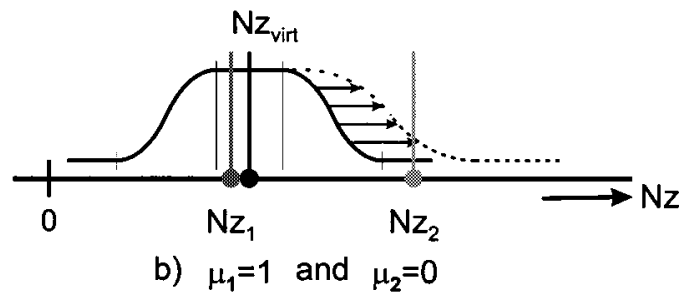

Fig. 12. Soft voting in the duplex operation. The virtual sensor output forms the center of a membership function, which is used to determine the membership degree of the hardware sensor outputs.

For each antecedent variable, two membership functions are defined. The rule-base therefore consists of $2^{3}=8$ rules. The degree of fulfillment of each of the rules is computed by taking the product of the membership degrees of each of the three premise terms

$$
\begin{aligned}
\mu_{1}= & \mu_{L O W}(M) \cdot \mu_{L O W}\left(Q_{c}\right) \cdot \mu_{L O W}(\alpha) \\
\mu_{2}= & \mu_{L O W}(M) \cdot \mu_{L O W}\left(Q_{c}\right) \cdot \mu_{H I G H}(\alpha) \\
& \vdots \\
\mu_{8}= & \mu_{H I G H}(M) \cdot \mu_{H I G H}\left(Q_{c}\right) \cdot \mu_{H I G H}(\alpha) .
\end{aligned}
$$

The output of the TS fuzzy model is the weighted output of the local linear models

$$
Z_{0}=\frac{\sum_{i=1}^{8} \mu_{i} \cdot Z_{0_{i}}}{\sum_{i=1}^{8} \mu_{i}} .
$$

Each aerodynamic derivative in (4) is described by a separate TS fuzzy model. The models, however, share a common set of membership functions which were obtained through fuzzy clustering and fine-tuned by genetic optimization [20]. More details on the design of the TS fuzzy model virtual sensor are given in [18]. 

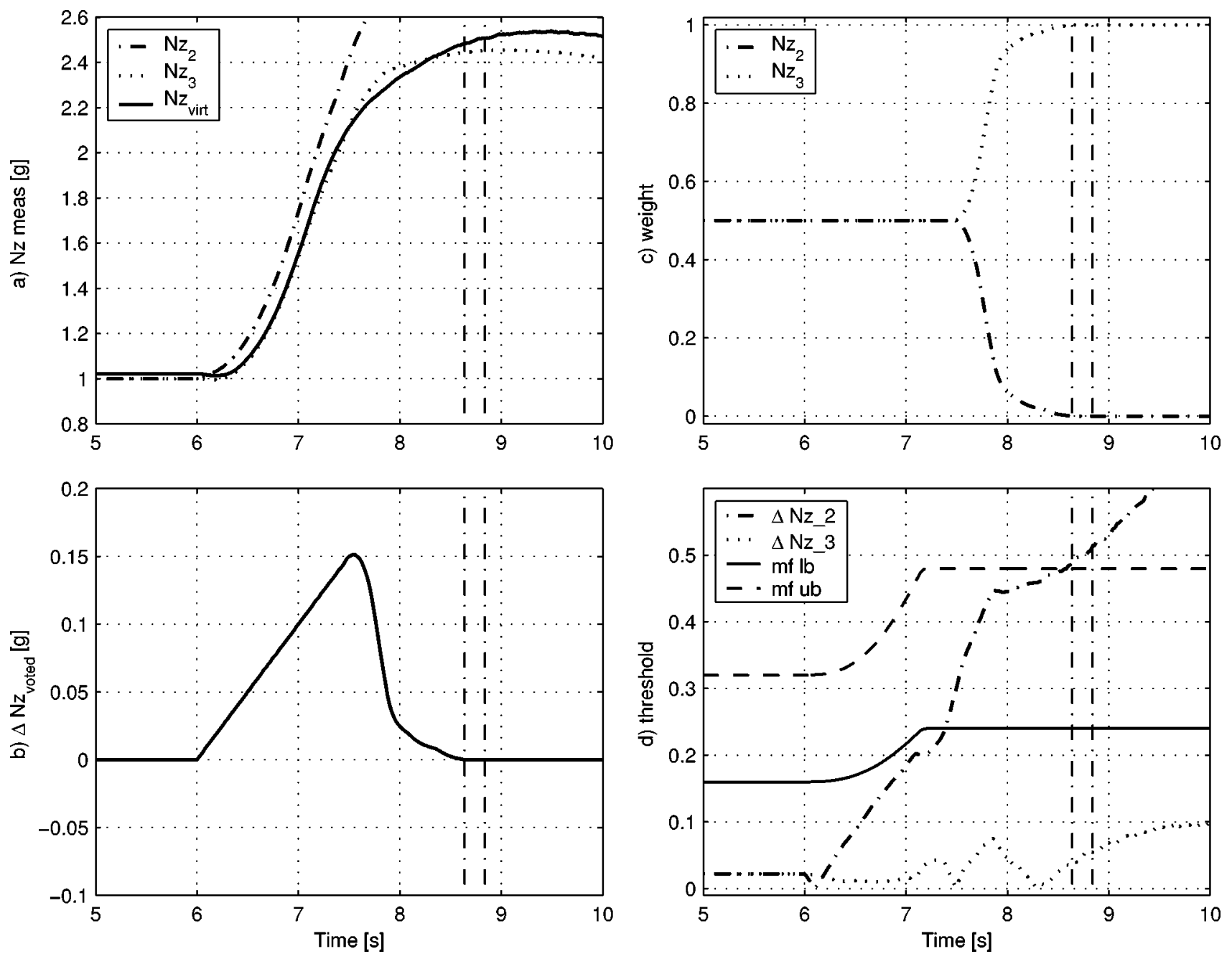

Fig. 13. Soft sensor management. Second drift failure of a normal acceleration sensor.

The above virtual sensor has been designed for different aircraft configurations, namely flaps/slats 00/00 (clean configuration), 12/25, 20/25 (take-off configuration), and 40/25 (landing configuration) degrees. The outputs of these four virtual sensors are then scheduled as a function of the flaps deflection $\delta_{f l}$, see Fig. 11.

With the virtual sensor, it is possible to identify the failed sensor even in the duplex operation.

As soon as the difference between the two remaining normal acceleration sensor signals exceeds a certain threshold, the virtual sensor serves a reference to compute the membership degree of both signals, by using a membership function centered around $N z_{\text {virtual }}$, see Fig. 12. In Fig. 12(a), the signal $N z_{2}$ is diverging from $N z_{\text {virtual }}$, and its membership degree $\mu_{2}$ with respect to the membership function of $N z_{\text {virtual }}$ is therefore less than one, namely $\mu_{2}=0.10$. The signal $N z_{1}$ and $N z_{\text {virtual }}$ are in agreement and the corresponding membership degree is therefore $\mu_{1}=1$. In Fig. 12(b), the signal $N z_{2}$ has further diverged from $N z_{\text {virtual }}$ and the corresponding membership degree is reduced to $\mu_{2}=0$. It should be stressed that in this case the virtual sensor only serves as an arbitrator and is not contributing to the consolidated signal.

\section{B. Dynamic Thresholds}

When using virtual sensors, the residuals resulting from the cross-comparison are more sensitive to uncertainties than in the case when only real sensors are used, especially with respect to unmodeled dynamics. Dynamic thresholds have been implemented to be able to deal with these uncertainties without compromising the fault detection sensitivity. Typically the estimation error of the normal acceleration virtual sensor is small during steady-state flight and increases during (aggressive) maneuvering. For this reason the support of the membership functions widens during maneuvering. In this way, a dynamic threshold is realized [see also Fig. 12(b)]. For the normal acceleration, the parameters of the membership function are adjusted as follows:

$$
\begin{aligned}
l b & =\min \left(l b_{\max }, l b_{\min }+C_{l b} \cdot N z_{\text {voted }}\right) \\
u b & =\min \left(u b_{\max }, u b_{\min }+C_{u b} \cdot N z_{\text {voted }}\right)
\end{aligned}
$$

where $l b$ and $u b$ denote the lower and upper bound of the membership function, respectively [see Fig. 12(a)], $C_{l b}$ and $C_{u b}$ are scaling factors. The dynamic thresholds have minimum $\left(l b_{\min }\right.$, $\left.u b_{\min }\right)$ and maximum $\left(l b_{\max }, u b_{\max }\right)$ values, where the maximum values are typically reached during maneuvering. The 

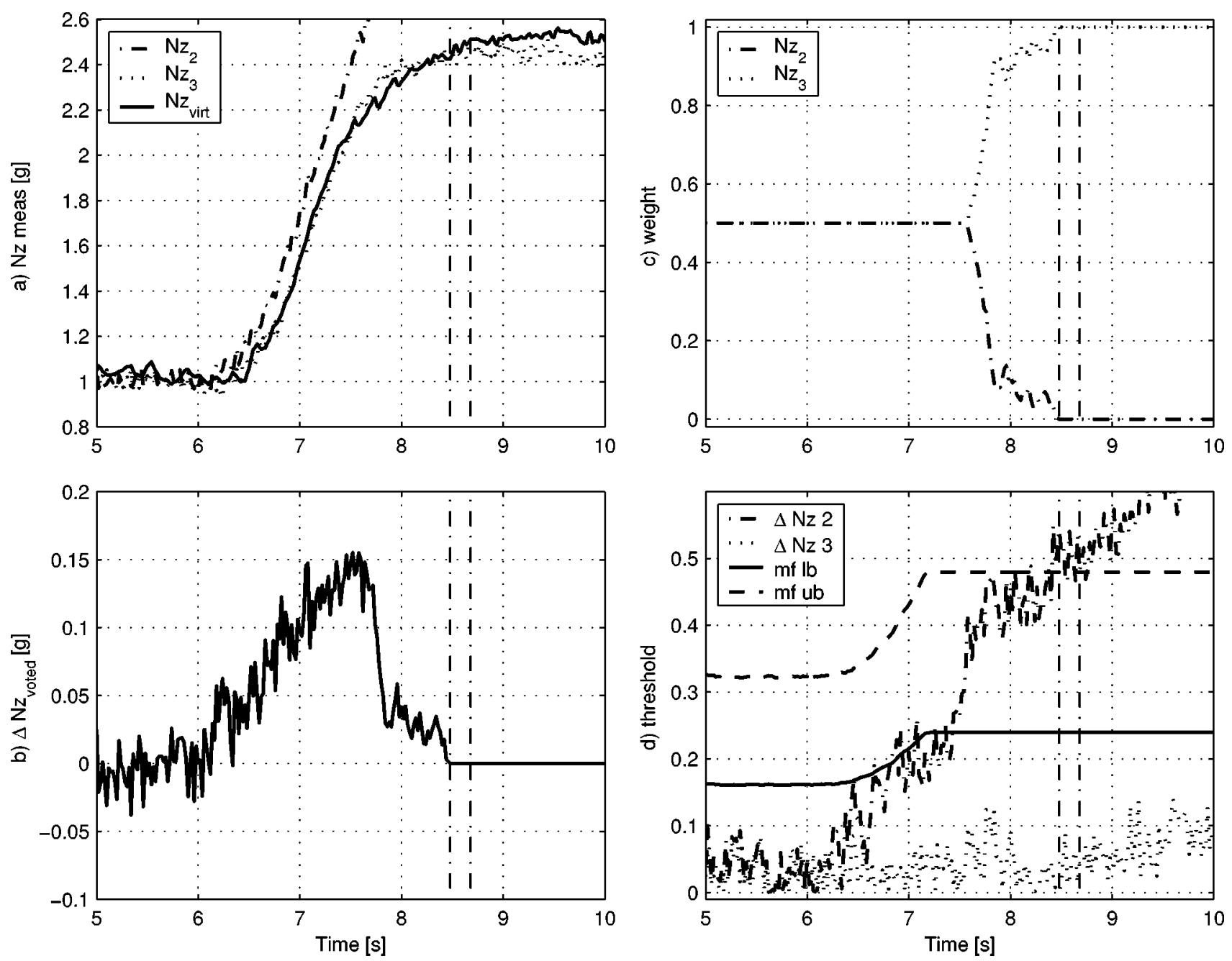

Fig. 14. Soft sensor management. Second drift failure of a normal acceleration sensor including sensor noise and severe turbulence.

dynamic thresholds enable acceptable sensitivity to failure detection, without increasing the false alarm rate, by keeping the soft threshold tight during steady-state flight only.

The dynamic thresholds are not enabled in the quadruplex and triplex operation, where the sensor management is based only on cross-comparison of real (hardware) sensors.

\section{Simulation Examples}

The virtual sensor enables the sensor management system to identify the failed sensor in duplex operation, see Fig. 13 for a drift failure of a second normal acceleration sensor. While signals $N z_{3}$ and $N z_{\text {virtual }}$ are in agreement, $N z_{2}$ starts to diverge from $N z_{\text {virtual }}$ [Fig. 13(a)]. In Fig. 13(b), the difference between the voted signal and the true normal acceleration $\Delta N z_{\text {voted }}$ is shown. Due to the drift, the voted signal diverges from the true normal acceleration until the weight of the failed sensor output is again reduced to zero [Fig. 13(c)]. The absolute differences between the sensor signals and the virtual sensor output $\left(\Delta N z_{2,3}\right)$ are illustrated in Fig. 13(d) together with the dynamic lower and upper bounds of the membership function connected to the virtual sensor signal. Here it is also illustrated that the dynamic thresholds indeed correlate with the estimation errors during maneuvering.
This scenario is repeated with sensor noise on all signals, including the inputs signals of the virtual sensor, and severe atmospheric turbulence; see Fig. 14. The soft sensor management system still performs well. The reason why in Fig. 14(b) the difference between the voted and true signal is equal to zero, even with sensor noise and severe turbulence, is because during the simulations $\mathrm{Nz}_{3}$ represents the true value.

Using the virtual sensor, the sensor management system is even capable of identifying a failure of the last available sensor, which is illustrated in Fig. 15 for a drift failure of the third normal acceleration sensor [Fig. 15(a)]. The monitor count is disengaged during simplex operation. As soon as the membership degree of $\mathrm{Nz}_{3}$ becomes equal to zero, the monitor count reaches the failure declaration value immediately [Fig. 15(d)] and the signal is no longer available. In Fig. 15(c), it is shown how the feedback path of the FCLs smoothly reconfigures to not using the normal acceleration signal.

It should be noted that even when a sensor failure is detected during simplex operation, it could be both the last available hardware sensor or the virtual sensor. In both cases the best strategy is to reconfigure to not using the normal acceleration signal. The implementation of the virtual sensor is not limited to the soft sensor management system, and could 

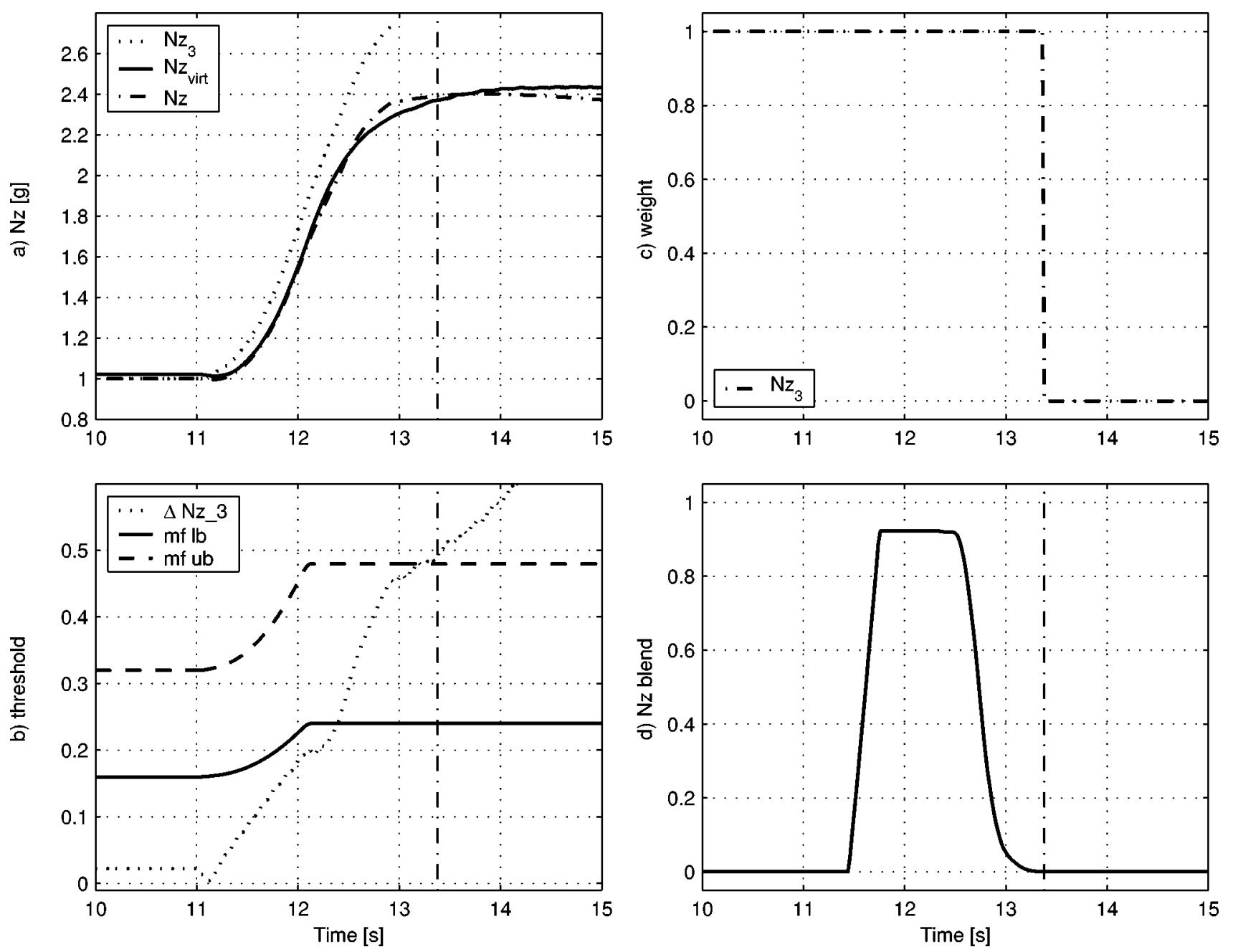

Fig. 15. Soft sensor management. Third drift failure of a normal acceleration sensor.

also be implemented in the conventional sensor management system.

\section{Flight Simulator Results}

The soft sensor management system, including the virtual sensor, has been successfully evaluated in pilot-in-the-loop simulations at the National Aerospace Laboratory (NLR) Research Flight Simulator.

Fig. 16 illustrates the result of one particular pilot-in-the-loop flight simulator test. During the simulations the data were recorded in batches containing mainly the insertion of the sensor failures and the more aggressive maneuvers performed by the pilot. The task of the test pilot was to try to find problems in the system and the virtual sensor, in the latter case by exiting the normal acceleration. Failures in two of the normal acceleration sensors were introduced at $t=95 \mathrm{~s}$ and $t=300 \mathrm{~s}$. The test pilot took his job very seriously, which can be concluded from the fact that the normal acceleration exceeded the maximum allowed value of $N z=2.5 \mathrm{~g}$ [Fig. 16(a)] and the bank angle reached a maximum value of $\phi=87 \mathrm{deg}$ [Fig. 16(b)]. Note that in normal flight the maximum bank angle will be limited to $\phi=33 \mathrm{deg}$. Even in these extreme situations, the soft sensor management system performed as expected.

\section{E. Discussion}

In the conventional sensor management system the consolidated signal is no longer available after a sensor failure in the duplex operation, even if one of the sensors is still healthy. The implementation of a virtual sensor makes it possible to monitor the last available hardware sensor. Since the virtual sensor is implemented in the software, the same safety level can be accomplished with less hardware. The cost reduction is more than the cost of the sensor itself, since it requires less supporting equipment and maintenance. Of course the development cost will increase because of the design of the virtual sensor.

Another issue concerning the virtual sensor is its dependence on a number of consolidated signals. Losing one of its inputs, it is most likely that the virtual sensor is lost as well. In that case the corresponding consolidated signal is lost after a sensor failure in duplex operation, since there is no longer an arbitrator available to isolate the failed sensor. However, the combined probability of losing one of the it inputs of the virtual sensor and getting down to a sensor failure in duplex operation for its corresponding signal is less then $10^{-9}$ and it is therefore "acceptable" that a catastrophic failure occurs in this case.

Virtual sensors are implemented to increase the capability of the available hardware sensors, or to be able to reduce the 

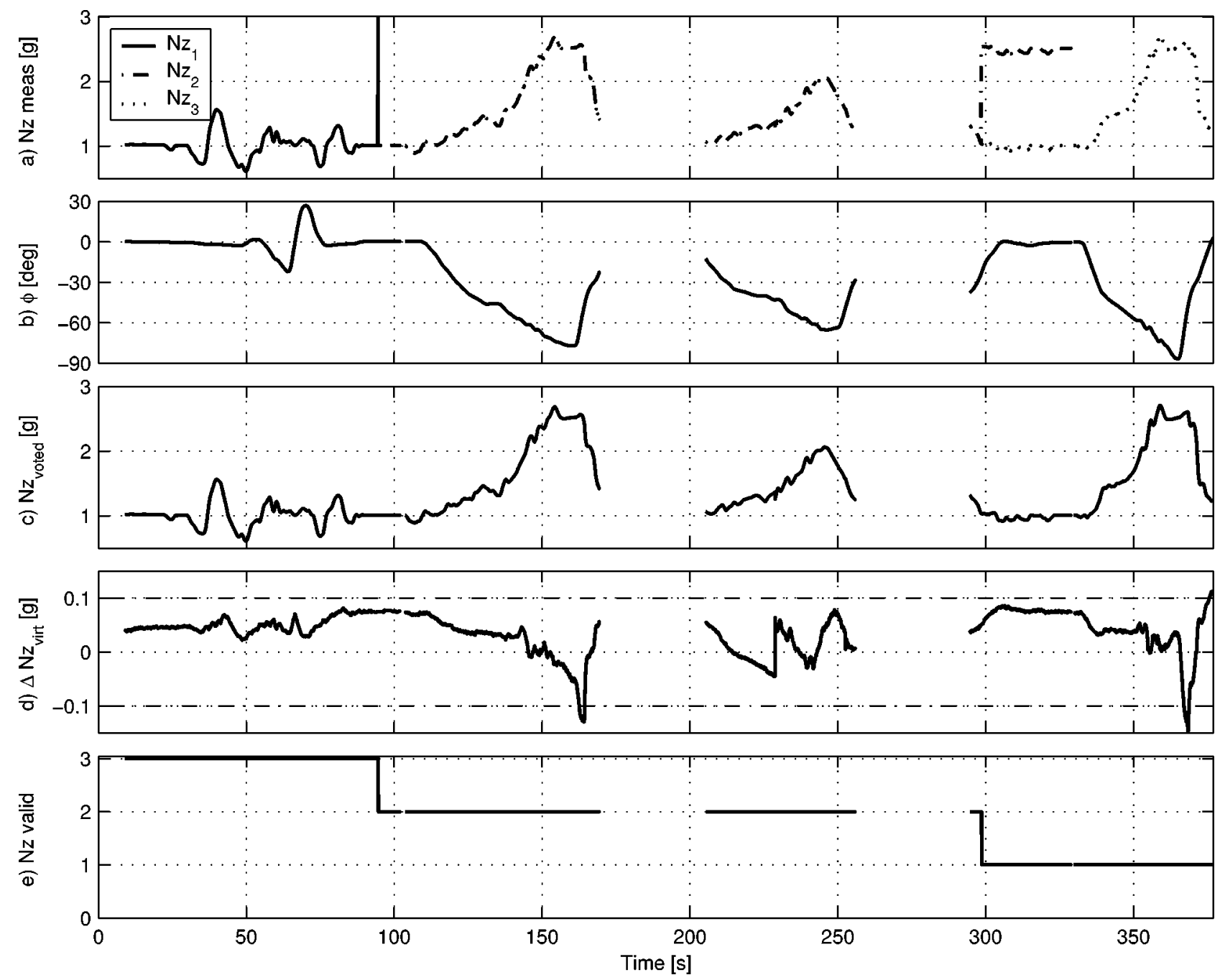

Fig. 16. Flight simulator test results.

number of hardware sensors without compromising the availability of the FCS.

\section{CONCLUSIONS}

Fuzzy logic techniques have been applied in the sensor management system, FCL reconfiguration, and the virtual sensor for normal acceleration. The improvement with respect to the conventional sensor management system is in the quality of the consolidated signal and results in a reduction of transients due to sensor failures. Furthermore, the virtual sensor increases the capability of the available hardware sensors, since it adds the ability to identify the failed sensor in the duplex operation and to detect a sensor failure in the simplex operation. This has been demonstrated by means of closed-loop simulation examples using a realistic aircraft model.

Final evaluation of the soft sensor management system and the TS fuzzy model based virtual sensor has taken place with pilot-in-the-loop simulations at the Research Flight Simulator of the National Aerospace Laboratory (NLR).

Future research will focus on the use of virtual sensors to identify multiple (equivalent) sensor failures and extension of the soft sensor management approach to actuator management.
Furthermore, tuning of the parameters of the membership functions, or dynamic thresholds, in a more structured way is still an open issue.

\section{ACKNOWLEDGMENT}

The authors thank the partners of this project for their contributions and useful comments.

\section{REFERENCES}

[1] K. Rosenberg, "FCS architecture definition (issue 1),", Deliverable 3.4, BE97-4098 ADFCS, 1998.

[2] P. M. Frank and T. Marcu, "Fuzzy logic control: Advances in applications," in Fuzzy Techniques in Fault Detection, Isolation and Diagnosis, H.B Verbruggen and R. Babuška, Eds. Singapore: Worl Scientific, 1999, vol. 23, Series in Robotics and Intelligent Systems, pp. $135-159$.

[3] H. Schneider and P. M. Frank, "Observer-based supervision and fault detection using nonlinear and fuzzy logic residual evaluation," IEEE Trans. Control Syst., vol. 4, pp. 274-282, 1996.

[4] R. J. Patton, P. M. Frank, and R. N. Clark, Fault Diagnosis in Dynamic Systems, Theory and Applications. New York: Prentice-Hall, 1989.

[5] R. J. Patton and J. Chen, "A review of parity space approaches to fault diagnosis applicable to aerospace systems," in Proc. AIAA Guidance Navigation and Control Conf., Hilton Head, SC, 1992, AIAA 92-4538, pp. $1-10$.

[6] R. Isermann, "Process fault detection based on modeling and estimation methods-A survey," Automatica, vol. 29, no. 4, pp. 815-835, 1984. 
[7] "Affordable digital fly-by-wire flight control system for small commercial aircraft," BE97-4098, 1997.

[8] M. B. Tischler, Advances in Aircraft Flight Control. New York: Taylor \& Francis, 1996.

[9] U. Ciniglio, F. Russo, and S. Scala, "Bare aircraft simulation tools: Model description and user manual," Deliverable 4.1, BE97-4098 ADFCS, 1998.

[10] D. McLean, Automatic Flight Control Systems. New York: PrenticeHall, 1990, International Series in Systems and Control Engineering.

[11] T. E. Menke and P. S. Maybeck, "Sensor/actuator failure detection in vista F-16 by multiple model adaptive estimation," IEEE Trans. Aerosp. Electron. Syst., vol. 31, no. 4, pp. 1218-1229, 1995.

[12] E. Y. Chow and A. S. Willsky, "Analytical redundancy and the design of robust failure detection systems," IEEE Trans. Automat. Contr., vol. AC-29, no. 7, pp. 603-614, 1984.

[13] S. M. Gopisetty and R. F. Stengel, "Detecting and identifying multiple failures in flight control systems," in 36th Aerospace Sciences Meeting \& Exhibit, Reno, NV, 1998, AIAA 98-4488.

[14] G. Schram, S. M. Gopisetty, and R. F. Stengel, "A fuzzy logic logicparity space approach to actuator failure detection and identification," in 36th Aerospace Sciences Meeting \& Exhibit, Reno, NV, 1998, AIAA 98-1014.

[15] P. M. Frank, "Fault diagnosis in dynamic systems using analytical and knowledge-based redundancy," Automatica, vol. 26, no. 3, pp. 459-474, 1990.

[16] R. R. Leal, P. Lane, and P. A. Payne, "Data fusion and artificial neural networks for biomass estimation," Proc. Inst. Elect. Eng. D, no. 2, pp. 69-72, 1997.

[17] E. L. Hanzevack, T. W. Long, C. M. Atkinson, and M. L. Traver, "Virtual sensors for spark ignition engines using neural networks," in Proc. ACC, vol. 1, Albuquerque, NM, 1997, pp. 669-673.

[18] M. Oosterom and R. Babuška, "Virtual sensor for fault detection and isolation in flight control systems-Fuzzy modeling approach," in Proc. IEEE Conf. Decision and Control, Sydney, Australia, 2000, pp. 2645-2650.

[19] T. Takagi and M. Sugeno, "Fuzzy identification of systems and its application to modeling and control," IEEE Trans. Syst., Man, Cybern., vol. SMC-15, no. 1, pp. 116-132, 1985.

[20] M. Setnes and J. A. Roubos, "Ga-fuzzy modeling and classification complexity and performance," IEEE Trans. Fuzzy Syst., vol. 8, pp. 509-522, Oct. 2000.

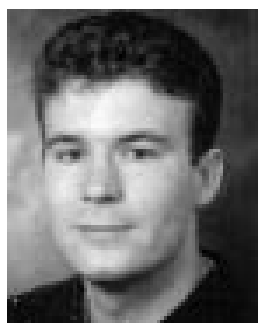

Marcel Oosterom (M'01) was born in 1974, Deurne, The Netherlands. He received the M.Sc. degree in aerospace engineering from the Delft University of Technology, Delft, The Netherlands, in 1998. He is currently pursuing the Ph.D. degree within the European ADFCS project at the Department of Control Systems Engineering, Faculty of Information Technology and Systems, Delft University of Technology. The topic of his research is (neuro-)fuzzy applications in flight control systems.

His research interests include flight control, gain scheduling, fault detection, isolation and reconfiguration, TS fuzzy modeling, and genetic algorithm optimization.

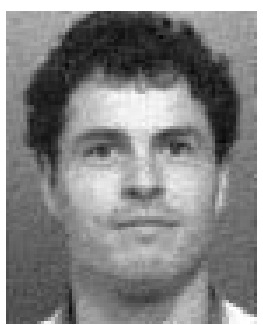

Robert Babuška received the M.Sc. degree in control engineering from the Czech Technical University, Prague, in 1990, and the Ph.D. degree from the Delft University of Technology, Delft, The Netherlands, in 1997.

Currently, he is a Professor at the Department of Control Systems Engineering, Faculty of Information Technology and Systems, Delft University of Technology. He is serving as an associate editor of Engineering Applications of Artificial Intelligence, and as an area editor of Fuzzy Sets and Systems. He has coauthored more than 30 journal papers and chapters in books and has published a research monograph Fuzzy Modeling for Control (Boston, MA: Kluwer 1998). His research interests include the use of fuzzy set techniques and neural networks in nonlinear system identification and control.

Dr. Babuška is serving as an associate editor of the IEEE TRANSACTIONS ON FUZZY SYSTEMS

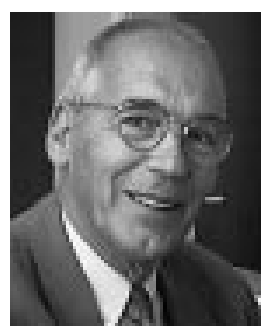

Henk B. Verbruggen received the M.Sc. degree in electrical engineering from the Delft University of Technology, Delft, The Netherlands, in 1963.

Since 1963, he has been Staff Member of the Control Engineering Laboratory, Electrical Engineering Department, Delft University of Technology. In 1980, he was appointed a Full Professor. His research interests include model-based predictive control, fuzzy logic and neural networks for modeling, control, fault detection, and controller reconfiguration. He is author and co-author of more than 200 publications. He is an associate editor of the IFAC journal Engineering Applications of AI and area editor of Fuzzy Sets and Systems.

Prof. Verbruggen served as chairman of the Coordinating Committee on Computer Control of IFAC. He was involved in a number of EC-sponsored research projects and working groups. 